\title{
All science as rigorous science: the principle of constructive mathematizability of any theory
}

\author{
Vasil Penchev, vasildinev@gmail.com \\ Bulgarian Academy of Sciences: Institute of Philosophy and Sociology: \\ Dept. of Logical Systems and Models
}

\begin{abstract}
A principle, according to which any scientific theory can be mathematized, is investigated. Social science, liberal arts, history, and philosophy are meant first of all. That kind of theory is presupposed to be a consistent text, which can be exhaustedly represented by a certain mathematical structure constructively. In thus used, the term "theory" includes all hypotheses as yet unconfirmed as already rejected. The investigation of the sketch of a possible proof of the principle demonstrates that it should be accepted rather a metamathematical axiom about the relation of mathematics and reality.
\end{abstract}

The main statement is formulated as follows: Any scientific theory admits isomorphism to some mathematical structure in a way constructive (that is not as a proof of "pure existence" in a mathematical sense).

Its investigation needs philosophical means. Husserl's phenomenology is what is used, and then the conception of "bracketing reality" is modelled to generalize Peano arithmetic in its relation to set theory in the foundation of mathematics. The obtained model is equivalent to the generalization of Peano arithmetic by means of replacing the axiom of induction with that of transfinite induction.

The sketch of the proof is organized in five steps: (1) a generalization of epoché; (2) involving transfinite induction in the transition between Peano arithmetic and set theory; (3) discussing the finiteness of Peano arithmetic; (4) applying transfinite induction to Peano arithmetic; (5) discussing an arithmetical model of reality.

Accepting or rejecting the principle, two kinds of mathematics appear differing from each other by its relation to reality. Accepting the principle, mathematics has to include reality within itself in a kind of Pythagoreanism. These two kinds are called in paper correspondingly Hilbert mathematics and Gödel mathematics. The sketch of the proof of the principle demonstrates that the generalization of Peano arithmetic as above can be interpreted as a model of Hilbert mathematics into Gödel mathematics therefore showing that the former is not less consistent than the latter, and the principle is an independent axiom.

\section{INTRODUCTION}

The research of first principles of all being conditioned the beginning of philosophy in Ancient Greece many millennia before the experimental science of the modern age. Euclid's geometry was built successfully for decades of centuries starting from a few axioms and postulates and deducing all rest statements in it as theorems logically. Thus philosophy
The present paper follows a pathway grounded on Husserl's phenomenology and "bracketing reality" to achieve the generalized arithmetic necessary for the principle to be founded in alternative ontology, in which there is no reality external to mathematics: reality is included within mathematics. That latter mathematics is able to self-found itself and can be called Hilbert mathematics in honour of Hilbert's program for self-founding mathematics on the base of arithmetic.

The principle of universal mathematizability is consistent to Hilbert mathematics, but not to Gödel mathematics. Consequently, its validity or rejection would resolve the problem which mathematics refers to our being; and vice versa: the choice between them for different reasons would confirm or refuse the principle as to the being.

An information interpretation of Hilbert mathematics is involved. It is a kind of ontology of information. The Schrödinger equation in quantum mechanics is involved to illustrate that ontology. Thus the problem which of the two mathematics is more relevant to our being (rather than reality for reality is external only to Gödel mathematics) is discussed again in a new way

A few directions for future work can be: a rigorous formal proof of the principle as an independent axiom; the further development of information ontology consistent to both kinds of mathematics, but much more natural for Hilbert mathematics; the development of the information interpretation of quantum mechanics as a mathematical one for information ontology and thus Hilbert mathematics; the description of consciousness in terms of information ontology.

Key words: axiom of choice; axiom of induction; axiom of transfinite induction; eidetic, phenomenological and transcendental reduction; epoché; Gödel mathematics; Hilbert mathematics; information; quantum mechanics, quantum information; phenomenology; principle of universal mathematizability

and geometry created a paradigm for constructing science from first principles, conserved until now.

The introduction of first principles independently of their relevance, from which the rest statements can be logically or otherwise deduced, completes the logical structure of the investigated area giving it the mathematical structure of lattice 
and thus, of both logic and ontology: Indeed, the first principles are the least element of the lattice, and the being as a whole or at least the investigated area is its biggest element.

Physics utilizing the method "by first principles" obtained a mathematical form. Nowadays, the boundary between physical theories and applied mathematics seems not to be different from that between mathematical structures and their interpretations. Some other sciences tried to follow the model of physics more or less successfully. However, other sciences, mainly in the scope of liberal arts, history, and philosophy implicitly or explicitly refuted the way of mathematization in principle.

On the contrary, philosophical phenomenology (Husserl's doctrine, first of all) establishes an inherent link between: (a) logic and mathematics; (b) philosophy; (c) psychology: The link relates the three by means a kind of transcendental idealism in the German philosophical tradition. Thus a bridge for transfer and reinterpretation between notions of psychology, logic and mathematics is created under the necessary condition for those concepts to be considered as philosophical as referred to that kind of transcendental subject.

One can question about the mathemazability of one (or any) scientific theory formally of that historical and conceptual background.

Statement: Any scientific theory admits isomorphism to some mathematical structure in a way constructive (that is not as a proof of "pure existence" in a mathematical sense).

Comments of the statement:

If any theory admits to be represented as the finite intension of a rather extended notion, the proof is trivial: Being finite, the intension can be always well-ordered to a single syllogism, the first element of which is interpretable as "first principles" (axioms): Those axioms generate a mathematical structure isomorphic to the theory at issue.

If one admits the axiom of choice, any intension can be well-ordered even being infinite. However, then the structure isomorphic to the theory would exist only "purely", which is practically useless.

In fact, any theory even as a description in liberal arts, philosophy or history is some finite text. This does not imply, though, that some finite extension corresponds to it for any text admits links to its context unlimitedly. Properly, this third case is what is worth to be proved mathematically.

A sketch of the proof:

Its essence consists in the approach of Husserl's phenomenology to be formalized and applied in both directions: to intension ("eidos", "phenomenon", intention) and to reality.

(1) One can introduce "epoché" both to "phenomenological" and to "eidetic reduction". As to the latter, it would mean the entire processes of removing one by one all free variables of the corresponding extension.

(2) The induction in Peano arithmetic is not sufficient to be obtained the finite intension of any real thing having infinitely many dimensions in its extension in general: One needs transfinite induction (or bar induction in intuitionist mathematics) for that purpose in the case of "eidetic reduction" or the "cut-elimination rule" in the case of "phenomenological reduction".
(3) Peano arithmetic is able to generate only finite numbers. Indeed, the following syllogism is obvious:

1 is finite.

Adding 1 to any finite number, one obtains a finite number.

Consequently, according to the axiom of induction, all numbers in Peano arithmetic are finite.

(4) One can complement Peano arithmetic to a complete model of reality adding to it a single bit, " $R$ ", interpretable as "infinity". That single bit is also interpretable as a second Peano arithmetic independent of the first one. The transition between the two Peano arithmetics in both directions needs transfinite induction or its equivalent. The completeness of Peano arithmetic is provable by transfinite induction.

(5) One can interpret that model of reality naturally in terms of Husserl's phenomenology if "epoché" is represented as removing " $R$ ": adding " $R$ " would be the reverse operation.

The statement, which is to be proved, can be called "principle of universal constructive mathematizibility".

The paper is organized as follows. Section 2 represents a few traits of philosophical phenomenology and first of all, of Husserl's phenomenology, which are essential to the problem of universal mathematization of science. Section 3 exhibits the main, explicit or implicit, philosophical and fundamental objections of liberal arts to universal mathematization. Section 4 demonstrates a complete enough proof of the statement. Section 5 offers a general philosophical interpretation of the principle of universal constructive mathemazation. Section 6 demonstrates by an example how the principle can be practically used for the choice of a relevant mathematical model. The last, $7^{\text {th }}$ Section summarizes and generalizes the paper to a few conclusions and directions for future work.

\section{THE PROBLEM OF UNIVERSAL MATHEMATIZATION OF SCIENCE AND HUSSERL'S PHENOMENOLOGY}

Set theory introduced the principle of abstraction [1] allowing of the generation of all elements of a set (interpretable as the extension of a notion) from a finite property featuring that set (as the definition or intension of the corresponding notion).

Still the initial development of set theory generated the axiom of choice [2] equivalent to the so-called well-ordering theorem [2] or "well-ordering principle" in contemporary terms. According to it, any set can be mapped one-to-one into some subset of the set of natural numbers and thus wellordered.

Semantics and semiotics of scientific theory elucidated it as a set of semantic units, which is well-ordered ("vocabulary of words") and thus designating the investigated area of objects ("things") in a way as ordered as possible, even well-ordered as an ideal by a one-to-one mapping between "words and things".

Scientific theory admits two kinds of exceptionally important formalizations: (i) as the intension of a rather extended notion, in which (i+ii) can be added well-ordering. The latter case corresponds to the deduction from first principles, which can be interpreted as the initial element of that well-ordering. 
This construction furthermore having the formal structure of logic is extended from the "beginning" of our knowledge in the first principles to the "end" itself of the being "by itself" (i.e. the biggest element of the lattice). Consequently, the first principles would complete our cognition as both logic and ontology.

The organization of knowledge in notions is much more economical, efficient and convenient for retaining, reproducing, transferring, and utilization. However, if the notion is as extended as a scientific theory, it is too huge even as a notion and needs a secondary compression to first principles and rules of deduction.

The organization by first principles being a secondary ordering refers to some description or definition of a notion, which can be a fiction or description of non-existing objects. Both notion about real things (or facts) and fiction can be equally well deduced from or compressed to first principles.

Thus that organization turns out to be especially relevant to mathematics.

Philosophical phenomenology starting from Brentano and Husserl introduced (or restored from scholastic philosophy) the conception about intentionality of consciousness [3-8]. Especially Husserl being a mathematician in education and early carrier linked that fundamental and definitive property of consciousness to the essence of mathematical cognition by means of the concept of "epoché" [7, 9]:

Indeed, mathematical cognition remains open the problem whether the described and investigated objects exist or not. In other words, mathematical cognition is invariant to and thus independent of the existence ("reality") or non-existence of its objects.

Thus attention turns out to be dual to the phenomenological "intention" [10-11] in a sense: It postulates its objects as real independently of whether they exist or not. So, the attention and intention constitutes a dual pair in dependence on whether the objects at issue are declared as real or not (here "not" does not mean for them to be declared as unreal or nonexisting, but that they might be real or unreal).

Then one can speak of "attention" as the reverse operation to "epoché": The latter takes or removes reality, and the former gives or adds reality. Thus attention being inherently linked to the problem of reality turns out to be a fundamental philosophical concept rather than only a psychological one. For example, if the operation of that philosophical "attention" is applied to any intention, one would obtain the corresponding "idea" or "eidos" (i.e. appearance as a whole) in a Platonic sense, i.e. as "reell".

Furthermore, "intention" has another counterpart, "intension" in logic, mathematics, epistemology, and cognitive science [10]. Intension is what is able to constitute unambiguously a separated unit such as a notion, set, image, or any unit of cognition by a finite definition, i.e. by a finite set of bound variables interpretable as the logical constant of that unit. An extension as the collection of objects, each of which satisfies the definition at issue, corresponds to any intension possibly as an empty one if the definition is contradictory. The collection may include as existing as nonexisting individuals.

One can introduce the concept of "attension" as to any unit enumerated above, e.g. as to a notion. It means both all individuals of the extension as existing and their wholeness as existing, too. Thus "attension" is relative to "intension" and "extension", on the one hand, and to the Platonic "idea" and "eidos", on the other hand. Furthermore, "attension" can be defined as the application of the "philosophical attention" to any explicit or implicit (e.g. contextual) intension.

Attension complements intension to the pair of both biggest and least element of the mathematical structure of lattice extended from the intention of consciousness to the idea therefore giving both logical and ontological structure of the notion or whatever else unit. That structure orders the extension in question in a potential taxonomy (i.e. classification of genera and species), the biggest element of which, i.e. the idea of the thing defined by the extension or even that thing itself or by itself, is generated just by the philosophical attention as the corresponding attension.

On the contrary, if the notion or unit is supplied as usual by any logical or ontological structure, thus its attension is implicitly certain, too.

The initial research of Husserl about the psychological foundation of arithmetic (1887-1891) [13-14] leaded him to opposite conclusion in the later "Logical Investigations" (1900-1901), namely that psychology (and further philosophy) should be underlain rather by logic and mathematics.

In fact, the initial base of that synthesis can be found even in Ancient Greece in Pythagoreanism, in the origin itself of philosophy, and a little later, in Plato's doctrine and Euclid's geometry. The German idealism including the subject and mind as a fundamental philosophical category had been what allowed of Husserl to add psychology in that huge synthesis.

The link in question is grounded in the way of cognition in logic and mathematics, philosophy, and the seen in thus psychology rather than in any reference to reality, to experienced or experimental data for the reality itself should be inferred in particular by the new approach of phenomenology: This suggests for reality not to be presupposed, but to be "bracketed" initially $[7,9]$.

Indeed, logic and mathematics do not connect the concept of truth, in their framework, to any confirmation by external reality. Therefore, they do not presuppose any reality, and their cognition is independent of reality as a hypothesis or premise. As to philosophy, it ought not to presuppose reality for the reality itself is its main problem ${ }^{1}$. At last, psychology should not be referred to reality as far as its object of research is just that being, which seems to be opposed to and thus separated from reality, namely mind and psychics ${ }^{2}$.

Thus logic \& mathematics, philosophy, and psychology need and would share a relevant method of research, which should be independent of the hypothesis (or axiom) of reality. In particular, that method cannot be experimental or ground on any experience in reality.

Logic and mathematics as the most advanced ones in that kind of cognition can suggest its extended model and interpretation where "intension" would correspond to "intention", and "extension" to some area of reality relevant to that intension at issue.

\footnotetext{
${ }^{1}$ Heidegger underlay the problem of being as a deeper one [15].

${ }^{2}$ Heidegger refuted this, the latter, and Husserl blamed him for "naturalization" [16].
} 
Then "attension" is the "extension" with reality added secondarily as far as reality cannot be presupposed in phenomenological research.

Husserl, both mathematician and philosopher, was who offered a new reading of transcendentalism, mathematical in essence. The transcendental might be understood as the collection of all possibilities therefore interpreting the "condition of possibility" in thus. Mathematics accepts consistency seen as the possibility of existence as mathematical existence as well. The collection of all possibilities might be defined as a certain invariant shared by all possibilities at issue, obtainable by "eidetic reduction", which is phenomenological in the sense of Husserl's psychology, or transcendental in his philosophy. One might say that eidetic, phenomenological and transcendental reduction are only different senses (or contexts) of one and the same meaning [9] mapping all possibilities of a kind into their shared invariant. Then Husserl's opposition of the phenomenological (transcendental) to the naturalistic might be further thought as the opposition of the set of all possibilities, defined by their invariant, to an arbitrary and therefore random element of the same set.

For example, the system of mind-brain unifies somehow both aspects allowing to be described as both mind (i.e. phenomenologically and transcendentally) and brain (i.e. naturalistically). One might even postulate that kind of duality as the essential feature of that system, necessary for its relevant definition. If that is the case, and Husserl's approach to the transcendental and naturalistic is used, one would need a certain equation of the transcendental and the naturalistic to define relevantly the system of mind-brain.

The interpretation of the mind-brain system as a quantum system $[17-19,11]$ satisfies the condition for an element of a set to be equated to the set, and therefore that of the reduction whether eidetic or phenomenological, or transcendental in Husserl's sense.

Quantum mechanics being only an exemplification and interpretation of a much more general set including it shares the same property, namely, the equivalence of a set to its element. Then, the term "quantum system" means it in the sense of both quantum mechanics and generalization definable by that equivalence of 'set' and 'element'.

No finite and constructive element can satisfy that kind of equivalence. Even more, that equivalence is interpretable as a version of Dedekind's definition of infinity [20], [21]. However, if the axiom of choice is attached, a finite, though unknown in principle, set equivalent one-to-one to each one infinite set should exist "purely" and mathematically, i.e. only possibly, but not ever actually. That paradoxical corollary is implied by Skolem's consideration [22] of the "relativeness of "set"' (1922). Thus infinity is decomposable to finiteness and randomness if randomness be equated to "pure" (never actual) possibility.

Then by interpreting in terms of mind-brain, a random element of the one half of that duality would correspond to each one element of the other. This is equivalent to the suggested by Niels Bohr conception about mind-brain complementarity [23], [24] as a generalization of complementarity in quantum mechanics.

One can interpret the "phenomena" in Husserl's sense as the existences ("existentia") of the "things themselves" or by themselves. Husserl rejected that approach as "naturalization" of his phenomenology [25-26]. Heidegger himself, though revising or developing far further Husserl's phenomenology, refuted to be an "existentialist" [27].

He tried to reinterpret Greek philosophy especially a few Pre-Socratics in that manner, in which the phenomenon (as "meaning it in itself by itself") might be identified as naïvely as wisely with the being (inseparable from the existence) of each certain thing.

The same approach of Heidegger penetrates, for example, his extended comment on a single fragment $(\mathrm{B}, 1)$ from Aristotle's Physics [29]. The part in question refers to the

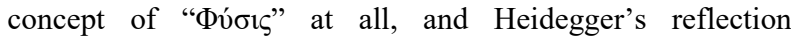
addresses the relation of that term in Greek philosophy and Aristotle's particularly to the modern European understanding of nature as opposed to both human being and technics.

Heidegger's way of interpretation merges the things and

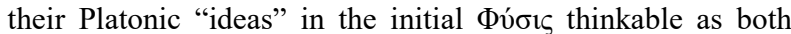
$\chi \alpha \dot{\alpha} \varsigma$ and $\dot{\alpha} \lambda \dot{\eta} \theta \varepsilon \dot{\varepsilon} \alpha$. Heidegger means the latter as that truth relevant to both Greek and his philosophy: $\dot{\alpha} \lambda \dot{\eta} \theta \varepsilon 1 \alpha$ is $\dot{\alpha}-$ $\lambda \eta \dot{\eta} \theta \varepsilon 1 \alpha$, i.e. the appearance at all from hiddenness as unhiddenness. That concept of truth is not underlain by any opposition to anything: it has not the form of the Latin adaequatio, the origin of which is often searched again in Aristotle.

Truth as $\dot{\alpha}-\lambda \dot{\eta} \theta \varepsilon 1 \alpha$ is phenomenon as appearance where being and existence are both yet and initially inseparable from each other. Thus truth as $\dot{\alpha}-\lambda \dot{\eta} \theta \varepsilon i \alpha$ is $\varphi v ́ \sigma 1 \varsigma$ at the same time. Nature is Truth before any opposition, particularly that of human being to nature.

Further, the Greek $\tau \dot{\varepsilon} \chi v \eta$ is seen in the same way rather than in the modern manner as creating something artificial, technical, which has not existed in a natural way, and even it might not exist in nature in principle: $\tau \dot{\varepsilon} \chi \vee \eta$ cannot be the modern technics at all.

On the contrary, $\tau \dot{\varepsilon} \chi v \eta$ means the hidden essence to be revealed, literally the veil to be removed, and thus truth to be seen: $\tau \dot{\varepsilon} \chi v \eta$ is not and cannot be opposed to $\varphi v ́ \sigma 1 \zeta$, it assists for the human beings to be able to observe the púris in an obvious way.

For example, a wooden chair reveals the strength and reliability of the tree, from which the chair has been made. That $\tau \dot{\varepsilon} \chi v \eta$ is not opposed furthermore to philosophy and poetry: It may be thought as another, namely material way of philosophizing or poeticizing.

Aristotle's $\dot{\varepsilon} \tau \varepsilon \varepsilon \lambda \dot{\varepsilon} \chi \varepsilon 1 \alpha$ is interpreted analogically and relatively to $\tau \dot{\varepsilon} \chi v \eta$ : it means the "essence to be given at the end" in Heidegger's interpretation, i.e. as the ultimate stage in the natural development. One may say that mankind and nature collaborate with each other by means correspondingly of $\tau \dot{\varepsilon} \chi v \eta$ and of natural development both sharing $\dot{\varepsilon} v \tau \varepsilon \lambda \dot{\varepsilon} \chi \varepsilon 1 \alpha$ as their essence.

What should be the Bedeutung (in the sense of Frege [30]) of metaphor?

It should be equated to an equivalent proposition, e.g. as in "sad moon" to "The moon is sad". However, that proposition is false in definition: otherwise it would not correspond to a metaphor. Furthermore, both false and metaphoric propositions share that they are wrong, but any wrong proposition is not a metaphor, even quite not: the metaphor is too rare and valuable because of that. 
Any proposition needs to be understood nevertheless whether it is true or false before any Bedeudung to be assigned to it. However, both false and metaphoric propositions have been understood in advance, and thus understanding cannot serve as a demarcation line between them. Anyway, the attitude to the understanding is different for each of them. It is a preliminary stage as to a nonmetaphoric proposition, after which it can acquire its Bedeutung of being false or not. The understanding is absolutely sufficient as to a metaphor or metaphoric proposition for being definitively false.

One can summarize: Understanding is the Bedeutung of metaphor, but not of false proposition.

The Bedeutung of a false proposition is namely "false", and any true proposition cannot be a metaphor or equivalent to a metaphor.

The next question is naturally to be the reversed one: A certain understanding is granted as Bedeutung. Which is the proposition true to that fact, e.g. to a metaphor?

A few preliminary notes are necessary. Human cognition can address as nature as culture. If the latter is the case, culture is put and opposed as a special kind of nature consisting of artefacts rather than facts and social events rather than phenomena. All of those kinds share the fact of understanding for no artefact might be created and no social event might happen without human understanding though they need not to be true to the facts or phenomena of nature. All culture shares understanding rather than adequacy to nature. Of course, some parts of culture, e.g. natural sciences can search for that adequacy, but they as well as the rest in culture share understanding fundamentally.

Consequently, the Bedeutung of metaphor as understanding is essential in all cognition of culture, but not in natural sciences, which add a new distinction of being either false or true as to the understood after understanding, though they share understanding as a necessary (but already extremely insufficient for them) condition being a part of human culture.

If hermeneutics is defined as the way (or method) of cognition of culture, it needs understanding as a fundamental concept, and the case study of metaphor may serve as a linguistic model or exemplification of hermeneutics.

Philosophical hermeneutics of Heidegger and Gadamer $[15,31]$ originated of Husserl's phenomenology [32], which suggested a new approach to the philosophical problem of consciousness. One might deduce the understanding in culture from the intentionality of consciousness for both "bracket" the adequacy to reality therefore addressing a new kind of reality, human reality, and a new kind of truth, which is not grounded on adequacy. It is called by Heidegger Alethea [15, 33] "unhiddeness" and it is applicable to the Bedeutung of metaphor as understanding. Indeed, anything which is understood comes out, exits from hiddenness, appears in "unhiddeness" sharing truth as Alethea nevertheless whether it is adequate to a certain external reality or not.

Then, which should be the proposition true to the fact that a certain understanding is granted as Bedeutung? The interpretation of "true" is ambiguous. If it is true as adequacy, that proposition should be equivalent to the understanding, e.g. as "The moon is sad" to "sad moon".

If it is true in the sense of Alethea, it should be an understanding of the understanding, a continuation in the same field of openness as unhiddeness (e.g. Heidegger's "Lichtung" [33]). In other words, it should be a new verse after the verse, which is just thus understood, following Heidegger's "Dichtung" [34], or a new text in the track of the text being understood just in thus, following Derrida's "trace" [35-36].

One can summarize that any understanding granted as Bedeutung can be described by one or more propositions adequate to the understanding as in humanities. Furthermore, the understanding can be understood by means of a new understanding, i.e. an interpretation, and thus kept, conserved and continued as a tradition (in Gadamer [31]).

Particularly, Ricœur's “true-and-false metaphor" [37], reminiscent of Schrödinger's "alive-and-dead cat" [38] rather essentially and fruitfully, can be interpreted consistently: Metaphor is true as understanding, but false as adequacy.

There is a fundamental philosophical problem about the relation of what is outside of consciousness to what is inside of consciousness. It implies that some boundaries of consciousness should exist in some sense to be the articulation of the former problem meaningful. On the contrary, the impossibility for that problem to be resolved is consistent to limitless consciousness as what is often interpreted God in theology.

A rather paradoxical equivalent to limitless consciousness was elaborated by Heidegger, but ascribed by him to Greeks: If Socrates's problem of human being had not been involved, therefor returning to pre-Socratics, all is being for there is not human being and thus any boundaries between the former and the latter do not exist. The concept itself of "Gegenstand" [29] as the opposing or being opposed to human being should be cancelled and erased.

Removing the human being is absolutely inacceptable for theology. Theology needs human being not less than God. So that kind of limitless consciousness as omnipresence being at the expense of cancelling the problem of human being is not equivalent to what theology means though that is consistent to limitlessness after removing the borders between what is inside and what is outside of consciousness. Heidegger in turn expressively rejected Sartre's "Existentialism is humanism" [39] as well as any link of his own doctrine (or "thinking") to existentialism and even "Sein und Zeit" later as far as it allowed of existentialist interpretations [28].

Consequently, theology or at least Christian theology conserves the problem of those borders but interprets it in terms of the relation of human being and God. Heidegger's term "ontotheology" [40] meant probably the same conservation, but as incoherent to his own thinking.

In fact, Heidegger's doctrine originated from Husserl's phenomenology rather than from Greek pre-Socratic philosophy immediately. Husserl's approach "bracketed" reality to reach the "phenomena" of consciousness. Thus, the question itself, about the borders of consciousness, turns out to be "bracketed", and the "phenomena" in Husserl's sense are invariant to the transformation "inside - outside" of consciousness. The question of those borders is rather meaningless as to the "phenomena" [41]. Then Heidegger's step is to interpret those invariant "phenomena" as being rather than as consciousness. Being is not existence, but invariant to existence and thus to human being. 
Husserl himself interpreted his own doctrine in different periods by means of Brentano's (or scholastic) intentionality [5], [42] of consciousness, of Descartes's apodictic consciousness [43], [44] and of Kant's transcendental consciousness [7] (and even of solipsism and "egology" [43] eventually interpreted as transcendental). Christian theology seems to be much more tolerant to Husserl than to Heidegger [46]. [47] One can generalize that Husserl remains always within consciousness, though expands it in a way to include the world. [48]

However, another interpretation of Husserl's phenomenology never shared by him himself might be grounded on mathematical cognition [49], [50] for he was a mathematician in education and early carrier. Indeed, mathematics is what has "bracketed" and always "brackets" reality being invariant to it. The mathematical abstractions are always "eidetic reductions" consistent to both reality and possibility. Further, eidetic reduction can be generalized to "phenomenological reduction" and "transcendental reduction" and include all contents of consciousness. Thus, first, psychology and then philosophy seem to be formulable as "strenge Wissenschaft" as mathematics. From that viewpoint, Husserl's doctrine might be classed as a form of neoPythagoreanism, say "transcendental Pythagoreanism".

In turn, that reading of Husserl's phenomenology can be applied to Heidegger's doctrine in a way to make the latter even still more radical. That thinking might be illustrated as a continuation in Heidegger's return to the origin of philosophy. Indeed, Heidegger did not include the Pythagoreans among the pre-Socratics [51]. His "homecoming" of philosophy reaches the Word of Heraclitus but not the Number of Pythagoras. Before Socrates's alleged overturn of being to human being, one might allege an earlier overturn of the Pythagorean Number to the Word of Heraclitus. Indeed, the Word and language presupposed understanding, the fundamental concept of philosophical or phenomenological hermeneutics invented or shared by Heidegger. If understanding is reduced further to human understanding, the reduction of being to human being is more than natural only after which the question of borders between the former and the latter might appear.

The further return in the origin of philosophy from the Word to the Number would mean in turn further desubjectification, but further dehumanization, too. The Word suggests understanding and thus admits human understanding, but the Number needs not either. It substitutes understanding by calculation. The tendency for philosophy to become an exact science leads to dehumanization in the final analysis if it is consistent enough. Indeed, the problem about the borders of consciousness disappear in thin air after that consistency, but human being, humanism, and then even understanding, and the being itself disappear in turn in the same way.

One can generalize that the problem of the borders of consciousness is among those problems which would be better not to be resolved rather then that they really cannot be resolved. The inconsistency of philosophy is fruitful. It allows of philosophy to be human philosophy just as understanding the human understanding, and 'being' to be restricted as the human being. Heidegger wrote about that "petitio principii" only which was able to allow of any science and even being to appear [29].
One can remind that Pythagoras and the Pythagoreans had kept their doctrine in both strict confidence and seclusion accessible only to the chosen ones [52]. Maybe they believed that it contents truth too destroying for mankind. Nowadays, we are able to reconstruct that too dangerous truth as consistent dehumanization to digitalization whether correctly or incorrectly.

\section{THE OBJECTIONS TO UNIVERSAL MATHEMAZATION}

There are a few main groups of explicit objections to mathematization of science along with implicit unacceptance and passive resistance, which can be extracted [53-62]:

(1) Mathemazation cannot be universal in principle. Mathematics creates only a more or less successful model (if any) which is fundamentally different from reality. The difference between any mathematical model and reality is qualitative rather than quantitative. Thus, that difference is beyond the range of mathematics in definition.

(2) Furthermore mathematization is not universal as a matter of fact. There are many sciences which do not admit any mathematization in principle. Some sciences such history or liberal arts study unique events, facts, or artefacts. They involve often human interpretation, values, and estimations. Mathematization is meaningless to them for it would deprive them of essence.

(3) Other sciences such as psychology or sociology study human factor whether in an individual or in society. Human factor does not allow mathematization in principle.

(4) Mathematization in many sciences, e.g. such as history, social sciences, biology, and even chemistry, etc. refer to unessential and auxiliary features or subdomains. Thus the core of those scientific disciplines is untouched by mathematization.

(5) Though possible in principle, mathematization in a fundamental sense is inconvenient for practical use. Engineer and applied sciences utilize often empirical and semiempirical functional dependences, which need not and are not connected to the correspondent fundamental sciences. The way to be deduced is often both unknown and never investigated. On the contrary, the attempts to be deduced simplified formulas for daily use from the fundamental equations met insurmountable issues, difficulties, obstacles and need groundless assumptions.

(6) Though mathematization is successful and practically applicable, its use needs immense calculations, which are beyond the present options even of supercomputers or are non-value-added because of the calculation cost.

(7) The mathematical models are unstable and deviate too considerably at slight deviations of the initial conditions (the "butterfly effect")

(8) Though the universal or partial mathematization might be possible in principle, it is dehumanizing and thus harmful.

The list does not pretend to be exhausted. Its claim is only to hint the huge volume of objections to mathematization, on the one hand, and to situate the present proof for universal constructive mathemazability of science on that background, on the other hand.

The proof does not refer to the group of objections (5), (6), (7), and (8) in any way. It touches the groups (2) and (3) only 
indirectly. Its meaning should be properly related to (1) and (4), but even then only partly.

Its sense in the background of those various objections may be described so:

As far as any science is exhibited in natural language, it admits exhausted mathematization by certain mathematical structures. The point is the extension from the rather obvious fact as to the exhibition in rigorous notions and consistently to the exhibition in any text in natural language thus covering all sciences (all sciences are represented in natural languages, but only a small part of them is already mathematized).

In other words, any text implies the option of mathematization rather than only any logically consistent text, in which the semantic units (such words) are well-defined as notions, and then well-ordered in syllogisms. This means that the difference between mathematics, called in Renaissance "Language of Nature" [53], and natural languages is neither fundamental nor even essential, but rather conventional.

Quantum mechanics was the scientific domain, which first met contextuality as to the language of nature. Before that, contextuality featured only human language and even it was considered as its disadvantage, which had to be "cured" by exact notions and definitions and a correct and rigorous logical order.

The overturn of common viewpoint for quantum mechanics was so drastic, unexpected, and sudden, that it was put "in quarantine", so that its "ridiculous conclusions" to be restricted to it itself and "classified" by complex technical calculations. The philosophical interpretation of its mathematical formalism based on the complex Hilbert space ceased gradually to be tolerated. The understanding of what in fact quantum stated was substituted by experimentally testable applications of it without explanations of what they should mean.

What forced quantum mechanics to so decisive steps was the necessity due to the existence of the fundamental natural Plank constant to find that kind of mathematical structure, which is invariant to the transformation between the discrete and continuous (usually even smooth) physical action. Classical mechanics is continuous (smooth). However, the Planck constant having the physical dimension of action, though exceptionally small, measures the action in quanta, i.e. by integers. The common explanation is that the actions in the Plank scale are discrete, but they seem as being continuous macroscopically for the exponent of the Planck constant is " -34 " to our daily measure units.

In fact, the mathematical formalism of quantum mechanics speaks otherwise. What it says is known as "wave - particle duality". It implies that quantum motion is both discrete and continuous (smooth) simultaneously. Any representation challenges common sense. As a corollary, quantum mechanics was forced to equate subjective and objective probability and even the possible and actual physically (the so-called probabilistic or Born interpretation of wave function [63]).

The "free will theorems" [64-65] can be deduced once both nonlocal formalism due to the complex Hilbert space and the local signaling for special relativity are granted simultaneously. Quantum mechanics is neither local nor nonlocal. It is both local and nonlocal.

As to the general problem of universal mathematization, two its conclusions are especially important:
(1) The necessity to be equated the discrete and continuous (smooth) to each other implies furthermore canceling the purely qualitative difference of model and reality. If quantum mechanics is true (as it seems to be), model and reality might coincide in particular, and the difference between them is both only quantitative sharing the mathematical formalism and qualitative as a mathematical formalism and as a separated reality simultaneously and in general [66]. That conclusion contradicts immediately to the objection (1) above.

(2) Quantum mechanics implies contextuality as to the language of nature [67]. Human language and the language of nature as what mathematics has accepted since the age of Galileo and Newton cannot be distinguished from each other for that identifier. Thus a huge obstacle for mathematics to penetrate all human knowledge is removed.

Still one objection, which can be added, refers to explicit Husserl's criticism to "Galilean mathematization" in one of the last works published in his lifetime, "The crisis of European sciences and transcendental philosophy; an introduction to phenomenology" [68], [56]. As if Husserl himself rejects universal mathematization even as one of the sources of "the crisis of European sciences". Consequently, any "mathematizing" interpretation of his phenomenology should be refused in turn, too.

Two main considerations on his criticism can be mentioned:

(1) Husserl criticized the universal substitution of qualities by quantities as naturalizing mathematization. Thus, what was meant was just Galilean mathematization rather than mathematization in general.

Mathematization had been done incorrectly since the age of Galileo being linked to naturalization and sensual qualities. One can suggest implicitly another mathematization grounded on "epoché" rather than on naturalization for mathematics really abstains from the statement of reality. Consequently, the adequate mathematization should be based on the approach of mathematics rather than on that strange assumption of reality external to mathematics.

(2) The criticism to "Galilean mathematization" should be seen as coherent and consistent to all other works of Husserl rather than opposed to them. He extended the approach of mathematics for abstaining of reality to those sciences such as psychology and philosophy, which should not presuppose reality, for the reality itself is their object and subject and a kind of petitio principii appeares.

\section{THE PROOF OF THE STATEMENT}

The statement is: Any scientific theory admits isomorphism to some mathematical structure in a way constructive (that is not as a proof of "pure existence" in a mathematical sense).

The statement needs a few preliminary elucidations.

(1) In fact, what will be proved is that any scientific theory, which is exhibited in natural language, admits that isomorphism constructively. One can object that a wider set of texts including the scientific theories allows of mathematization. This seems to be namely so. However, the question about which texts are scientific theories [69] is rather difficult to be answered unambiguously.

Nevertheless, any scientific theory is a text otherwise a counterexample might be demonstrated. This means that the 
following arguments as a sketch of proof can be considered as an implicit definition about that wider set of texts including the scientific theories: those texts which ate a true part of more extended texts and thus refer to nonempty contexts. That kind of definition forbids only the consideration of any text claiming to be scientific as universal, i.e. without any context. It is similar to Zermelo's way out from the paradoxes in set theory [70]: any set is a true subset of another, and if not it is not a set. No scientific text is a universal dogma, which is always valid independently of its context and therefore corresponds to Poper's criterion [71] not to be "metaphysical".

(2) The sketch of the proof can be divided into three cases, which are rather unequal in a mathematical sense. The first two ones are almost obvious and they need not to be proved, or in other words, their proof is "trivial". Nevertheless, their interpretation including philosophical interpretation is not less important than that of the third case properly needing a mathematical proof.

(3) The term 'theory' is used rather in an internal sense than in an external sense. This means that the question about their confirmation in reality by experience or experiments is "bracketed" in a phenomenological manner. Consequently, the term should include also hypotheses still yet neither corroborated nor rejected as well as even old, already refused hypotheses.

(4) The first case is that of a classical theory exhibited consistently, i.e. by well-defined notions well-ordered in syllogisms. A mathematical theory deduced from a complete and consistent set of axiom can be considered as the "ideal case" (in the sense of Max Weber's "idealtypus" [72]) of that first case. Of course, a mathematical theory does not need any additional mathematization for it is mathematized in definition. All theories of the first case are differed slightly from their ideal case.

The term "slightly" as it is used above needs a more certain justification. "Slightly" is used in relation to the degree of separation of the theoretical text from its context as well as to those degrees as to the notions or postulates (principles) inside the text itself. The ideal case corresponds to absolute separation. One might argue even that the ideal case of scientific theory is not a scientific theory for it does not have any context in fact after it can be absolutely separated from its context. However, this does not contradict to Weber's concept of idealtypus [72].

This means that the text is a whole in isolation from and independent of its context. The same refers to its notions to all rest ones. If the text at issue is taken out of its natural context, in which it has appeared initially, and incorporated to other relevant context, its meaning will not be changed.

In fact, that in the sense of absolute independence can be hardly referred even to a present mathematical theory. As the discussion of the third case will show, those absolutely "acontextual" theories do not exist in principle just as any quantum system, what any physical entities is, cannot be separated absolutely from its environment in virtue of the Kochen - Specker theorem [67]. The meant "ideal case" is unachievable by any real theory even by a mathematical one.

The term "slightly" can be anyway described as to practical use by the model of a mathematical theory, in which can miss some notions or axioms as well as the proof of completeness and consistency including the mutual independence of its axioms. Euclid's geometry can be a great example of using "slightly" relevantly. Indeed, it had been the standard of how a scientific theory should look like throughout many centuries and even millennia. That standard turns out to be too high even nowadays for many sciences out of the scope of the exact ones.

Besides being preliminary almost mathematized at least implicitly as slightly differing from its ideal type, any theory falling within the first case can be considered as the intension of an extended notion or as the definition of that notion. Thus it can be directly defined in terms of information in the sense of Piers [73]: any theory, of course being furthermore a "sign", should not be "an icon" conveying information about everything or nothing [74]. The applicability of Peirce's "information" is quite natural after its definition of "state of information" [74] almost coincides with a result after Husserl's eidetic reduction.

Though its explicit extension can comprise infinitely many individuals, the intension can be always chosen to be finite. This corresponds to the principle of abstraction [1] as to set theory: any infinite set can be described by a relevant property expressible finitely.

Being representable as a set, it is both unambiguously distinguished from its environment, and its elements from each other. One can say that the first case is well-modelable in terms of set theory. This is not surprising at all for one of the natural contexts, in which the set theory itself appeared, was that of that kind of theories.

(5) Unlike the first case, the second case does not offer historical and even only natural interpretation. It should be involved for the distinction of the constructive from nonconstructive mathematization.

If both, an arbitrary text and the axiom of choice are given, a finite set as those in the first case and equivalent to the text has to exist but only "purely and mathematically". This means that finite set in question cannot be demonstrated in principle as one and the same in any way. That pure existence as any pure existence in mathematics is useless for practice. That text is mathematizable in principle, but nobody knows how. Even the corresponding equivalent mathematical form might be provably inexpressible explicitly.

Nevertheless, that second case hints the method, in which the practically significant third case might be researched and proved.

That existence in the second case is grounded on Skolem's relativity of the concept of set (1922) after the axiom of choice [22], also known as Skolem's paradox.

Indeed, let us admit that any text doubtlessly being finite anyway can be represented as a certain set though infinite in general. (That assumption will be discussed and justified a little later.) After the axiom of choice is given, it can be well-ordered and therefore enumerated, i.e. a natural number can be attached unambiguously to each one element of the set.

Peano arithmetic [75] implies that any natural number is finite. Indeed, 1 is finite; adding 1 to any finite number, one obtains a finite number again; then, all natural numbers are finite according to the axiom of induction. One can obtain infinite natural numbers only if the existence of at least one infinite natural number is postulated. However, there is not such an axiom, at least among Peano's. 
Then, the set in question, after having been well-ordered, is furthermore enumerated by successive finite numbers. It is possible only if it itself is a finite set. Summarizing, the axiom of choice and Peano arithmetic imply that any infinite set can be enumerated and therefore mapped one-to-one by a certain finite set equivalent to a certain initial segment of natural numbers. It was just still Thoralf Skolem (1922) who pointed out expressively that concept of 'set' is relative even to the distinction "finiteness - infinity" [22] after the two assumptions.

However, that corresponding finite set exists only purely: it cannot be demonstrated explicitly ever in principle once the axiom of choice is utilized.

That pure existence might be equated to the random choice of an initial segment of natural numbers. That additional assumption seems to be consistent to the first two ones after one of them is just the axiom of choice [76]. Furthermore, though the corresponding finite set is randomly chosen after each one experiment for that set to be attached to an infinite set, one can admit that the probability distribution for some finite set to be attached to a certain infinite set is unambiguously determined.

The latter two additional assumptions though unnecessary for the correspondence between finite and infinite sets (the former two ones are sufficient) allow of unambiguously linking of a probability distribution to each infinite set [77].

The initial assumption that any text can be represented as an equivalent set infinite in general will be discussed now. If it is granted, one can trace the pathway for universal though yet nonconstructive mathematization. However, it seems just like petitio principii for set theory is the ground of mathematics.

If any theory being a text in general is declared to be a set, this presupposes already the principle of universal mathematization.

The same consideration refers to the discussion of the first case, too. There, any theory differs slightly from the ideal type, which is postulated to be that of mathematical theory. If it is granted, it seems to be obvious that theory should be mathematizable.

The same kind of petitio principii will be referable after the discussion of the third case a little later. Then, the question about what is the meaning and value of such a principle of universal mathemazibility and its proof is natural.

If one presupposes that any scientific theory is mathematizable, it turns out to be just that.

Its meaning and value might be interpreted as follows. One can postulate and therefore choose that universal mathemazibility or not. If the former is the case, there is a certain relevant mathematical structure, which can be found.

Nonetheless, one can choose not to mathematize the theory at issue. Consequently, the proof is able only to demonstrate that principle is independent and eventually consistent to human cognition and its ground.

One can compare the situation with the discovery of nonEuclidean geometry [78]. One can accept a certain negation of Euclid's fifth postulate searching for any contradiction and therefore for a proof of the fifth postulate by reduction ad absurdum. However, that contradiction is not found. Even more, one can construct a model of non-Euclidean geometry inside in the Euclidean geometry itself. Consequently, the consistency of Euclidean geometry implies the consistency of non-Euclidean geometry, too.

Analogically, the principle of mathematizability says: the consistency of any scientific theory implies the consistency of its mathematized build; the choices to mathematize or not are both acceptable and equally consistent.

Nobody is forced to mathematize. This is a free choice, which is justifiable

(6) The third case, the properly mathematically interesting one, refers to the addition of constructiveness in a consistent way to the second case discussed just above. This needs Husserl's fundamental and philosophical considerations about reality and "phenomenon" in his sense explicitly. Furthermore, one has to interpret them in terms of Peano arithmetic and apply to set theory.

The introduction of the kinds of reductions in his sense is crucial. Those are: "eidetic reduction", "phenomenological reduction", and "transcendental reduction". He declared that all the three share one and the same meaning ${ }^{3}$ utilized in three different contexts: correspondingly mathematics, psychology and philosophy. Those three ones share furthermore a special attitude to reality, which is not the natural one, for it itself as a whole is a subject of research. Consequently, its existence cannot be presupposed as mankind and all other sciences have always done. That "natural attitude" is to be "bracketed" as to the range of each of them. Thus both psychology and philosophy might be transformed into "rigorous sciences" what mathematics is.

The present paper can be interpreted as an approach to the generalization of Husserl's idea from the scope of psychology and philosophy to that including any scientific theory. The latter can be always mathematized in a constructive way and thus transformed into a "rigorous scientific theory" if one wishes.

Husserl's terms "epoché" and "bracketing" [7, 9] serve to express that nonstandard attitude to reality. It is not rejected if one utilizes those terms. It is neither rejected nor accepted. The problem of reality is remained open to be possible its research by scientific methods. After that problem is presupposedly open, one can investigate what remains just by a kind of reduction certain according to the corresponding domain: mathematics, psychology, or philosophy. Until Husserl, the method had been explicitly developed only in mathematics.

Its essence is given in Husserl's manner of thought by the concept of eidetic reduction [7], [79]. One can remove one by one the properties of anything questioning after each one removing whether the investigated thing continues to be the same. If yes, the process continues by the next removing, and if not, the last removing is cancelled, i.e. the process is returned to the immediately antecedent state, and the property at issue is enlisted into the eidos of the thing ${ }^{4}$. Then the process continues as initially.

The most essential feature of that algorithmic procedure is its constructiveness as to the present consideration. One will

\footnotetext{
3 Here "meaning" is used as Frege's "Bedeutung" [30], but not as reference or referent. The latter is inacceptable for reality should not be presupposed.

${ }^{4}$ Which corresponds to the state of information of that thing in Peirce [74].
} 
obtain the eidos of the investigated thing as an ultimate and unambiguous result.

That thing will turn out to be axiomatized as to mathematics. It can be exhaustedly restored deductively from its axioms and any of its properties will be proved as a theorem.

However, a finite set of properties can distinguish only a finite set of individuals. Consequently, if the set of individuals is infinite, as usual in mathematics, it needs a set of property, which cannot be finite, for the set of all subsets of any finite set is finite in turn.

Furthermore, if the above procedure is represented by Peano arithmetic, there is no guarantee that it can be accomplished. The thing has an infinite set of properties for it is defined in set theory. However, Peano arithmetic does not include infinite natural numbers for the axiom of induction, as this is demonstrated above. The procedure is representable in Paeno arithmetic, but it refers to set theory. The former does not include infinity, and the latter does. The only consistent way out is that the procedure will not finish to be able to reconcile finiteness to infinity.

That way out is not relevant as to the discussed procedure for the eidos of the anything to be obtained.

One needs to postulate the existence of at least one infinite natural number and therefore to generalize Peano arithmetic and axioms. The same postulate is historically equivalently realized by the concept and axiom of transfinite induction [80] generalizing those of induction in Peano arithmetic [81].

Indeed, the transfinite induction replaced that of induction in Peano arithmetic allows of resolving the problem of reconciling arithmetic, set theory, and the discussed procedure for obtaining eidos consistently. The transfinite induction implies the existence of natural numbers, which are not finite as well as that the procedure referring to an infinite set of properties is able to finish.

The case where the procedure cannot finish corresponds to Gödel's "undecidable statements" (1931) [82] for they appear also on the boundary between Peano arithmetic and set theory. Indeed, the former does not admit infinity unlike the latter, and the only way to reconciled consistently to each other is the existence of those statement, the resolving of which cannot finish.

On the contrary, they disappear into thin air after transfinite induction has been applied as in Gentzen's proof (1936) [80, 83], which is also coherent with the consideration just above.

Husserl's approach for "bracketing reality" also leads to an equivalent of transfinite induction once it is interpreted in terms of arithmetic and set theory. Here is how:

There are two disjunctive options: to "bracket reality" or not; this corresponds to adding a single bit with two values: "consciousness" and "reality". If that bit has not been added at all, this is a "phenomenon", which is equally relevant to both consciousness and reality. Of course, that extremely simplified scheme is necessary only for the interpretation in terms of arithmetic, which by itself is too simple.

One can summarize that only a single bit to be always addable is sufficient. However, the so-called function successor within the framework of Peano arithmetic as if guarantees the same, and this is not enough. In fact, the additional bit, meaning the bracketing of reality as a whole, should refer to the Peano arithmetic itself as a whole. So, the additional bit does not double any natural number, but doubles the set of all natural numbers given by the Peano axioms and thus generates a second Peano arithmetic absolutely independent of the first one though identical to it.

Then, the latter can be interpreted as the "arithmetic of infinity". The Peano axiom of induction is absolutely enough within each of them, but irrelevant as to the transition between them. That transition needs transfinite induction.

As the elucidated link from the additional bit to transfinite induction is a chain of equivalencies, the reversion from transfinite induction to an additional bit in the sense above is valid, too.

This accomplishes the sketch of the proof in the third case.

That arithmetical model of reality also excludes the Gödel undecidable statements in Gentzen's manner [80].

One can utilize the term "Gödel mathematic" if the principle of universal mathematizability is not accepted and there are statements which cannot be resolved. They would correspond to non-mathematizible scientific theories.

For the Hilbert program [84] of how mathematics might self-found itself, one can call that kind of mathematic, where the principle of universal mathematizablity is valid, "Hilbert mathematic". There are not undecidable statements or nonmathematizable scientific theories in it for Gentzen's proof $[80,83]$.

The ontological status of the two kinds of mathematics is quite different:

The Gödel mathematics corresponds to Husserl's "natural attitude" to reality. It exists as external and qualitatively different from mathematics. It is consistent to the most philosophies of mathematics.

The Hilbert mathematics would correspond to Husserl's "epoché" to reality. It is able to include reality within itself. The kinds of Pythagoreanism would be the most relevant to it.

The principle of universal mathematizability is consistent to Hilbert mathematics. As in relation to the principle, anyone is free to choose between Gödel mathematics and Hulbert mathematics. Whatever would be the choice both alternatives are consistent and justifiable.

\section{THE PHILOSOPHICAL INTERPRETATION OF THE PRINCIPLE}

The philosophical interpretation of the principle was touched many times above in the corresponding link to concrete questions.

There is still one way of interpretation if one would like to elucidate which mathematics is more relevant to our being ${ }^{5}$ : whether Gödel mathematics or Hilbert mathematics. It should deduce the "elements of being" in each case.

The answer as to Gödel mathematics is well investigated: mathematics and reality does not share common elements: the

\footnotetext{
5 Here is used the term "being" in a fundamental and philosophical sense for the usual terms of that kind, such as "the world" or "reality", make sense only in the context of the first alternative, that of Gödel mathematics. So, their utilization is irrelevant if the problem at issue is to be discussed without bias.

6 Those can be thought as a kind of reminiscence of Einstein, Podolsky, Rosen's "element of reality" [85] in a quite different, and even opposite context.
} 
ones, in mathematics, are ideal and the others, in reality, right real. Different degree of correspondence between them as well as various hypotheses or theories can be admitted, however they are always two fundamentally different types even in the cases where the one type is suggested to be reducible to the other.

As to Hilbert mathematics, the problem is not yet researched. Here is an approach outlined:

The three cases in the sketch of proof above correspond to finite set, infinite set non-constructively, and infinite set constructively. The first and third cases can be unified as constructive (any finite set is trivially constructive), and opposed to the second one, right non-constructive.

The sketched way of proof in the case of constructiveness refers to arithmetic whether the Peano one (the first case) or the one generalized by transfinite induction (and equivalent to two Peano aritmetics).

Discussing the second case above, that of nonconstructiveness, two last assumptions were made at the end:

(1) Pure existence might be equated to the random choice of an initial segment of natural numbers.

(2) Probability distribution for some finite set to be attached to a certain infinite set features unambiguously the certain infinite set.

They trace an alternative way for that constructiveness to infinite sets, which does not need arithmetic, the well-ordering principle and the axiom of choice in the final analysis, though the way was above pioneered by means of the latter two ones.

Its essence consists in "bracketing" finiteness as follows:

The transition from any infinite set to a randomly corresponding finite initial segment of the natural numbers needs indeed the axiom of choice. However, once the corresponding probability distribution is constructed, it can be postulated as corresponding to the infinite set at issue. Then the transition from one infinite set to any other one is an operator of probability distributions, which does not need the axiom of choice at all.

The relation of infinity to finiteness, though being fundamentally random, is involved indirectly, by its ultimate result of a certain probability distribution, the transformation of which is not random anymore in turn. This is meant as "bracketing finiteness": it differs from ignoring finiteness. If the latter was the case, infinity might be treated as a second finiteness as after exploring a second Peano arithmetic for transfinite induction, but indistinguishable from the first one.

One remains in the transformations of only infinite sets, however determinable unambiguously to the finiteness as a whole right by the probability distributions.

So, two ways of representing infinity sets may be distinguished accordingly. The one is grounded on probability distributions, the other on arithmetic generalized relevantly.

One can suggest that both approaches should be equivalent to each other, and even that the one can be referred to "reality" and the other to "mathematics" if need be for comparing with "Gödel mathematics".

In turn, the utilization of probability distributions can significantly facilitate elaborating mathematized builds of scientific theories if the above equivalence holds.

If any well-confirmed and fundamental scientific fact can be considered as an interpretation of that equivalence, it will be in favor of the hypothesis of Hilbert mathematics as to our being.

At last, that universal kind of elements shared by both mathematics and reality in Hilbert mathematics coincides with the concept and quantity of information.

Indeed, Peano arithmetic can be interpreted as a universal Turing machine processing a tape consisting of bits, i.e. of the elementary units of information. Any real computer can be modeled as a Turing machine, which "tape" is always finite if the computer can finish its work. This corresponds to the case of Peano arithmetic.

Its generalization as above for transfinite induction needs a corresponding generalization of Turing machine: the introduction of results calculable by transfinite "tapes" is sufficient. No real computer existing until now can be modeled as having that "transfinite tape"

The case of probability distributions can be represented as a quantum Turing machine processing qubits rather than bits and a tape, in which all bits are replaced by qubits. A qubit is defined in quantum mechanics and theory of quantum information as the normed superposition of two orthogonal subspaces of the complex Hilbert space. Thus, the complex Hilbert space itself can be represented as the tape of the quantum computer, in which different wave functions (interpretable as the states of physical systems) are processed.

A qubit can be considered as that generalization of a bit, which refers to an infinite series or to an infinite set of alternatives just as a standard bit is defined as the choice between two equally probable alternatives.

The corresponding kind of information is quantum information. Indeed, the wave functions processed by the quantum Turing machine can be interpreted as the characteristic functions of those probability distributions specifying the corresponding infinite sets at issue.

Consequently, a kind of information is what is (i.e. the being) in all cases. Information can be represented as a finite series of bits, as a transfinite series of bits, or as a series of qubits.

Where Hilbert mathematics is valid, all is information.

\section{AN EXAMPLE OF USE}

As this is demonstrated above, there are three "kinds" of information and their corresponding units: bits, transfinite bits (i.e. bits in transfinite positions or in other words, those corresponding to the second Peano arithmetic), and qubits. The option of being equated to each other is natural for Hilbert mathematics, but anyway consistent with Gödel mathematics. The difference between the two cases is rather philosophical and interpretational than formal:

If Hilbert mathematics is the case, information is one single, and the kinds of information are identical in definitions though expressed differently. However, the kinds of information are different in definition as to Gödel mathematics, but they might be equated to each other occasionally.

Consequently, any corroboration in favour of each of them needs the distinction whether the case of information equation is fundamental or partial and thus depends again on the a priori philosophical framework of interpretation. 
For example, if a fundamental equation in quantum mechanics such as the Schrödinger equation is interpreted as an information equation successfully, the question whether it is in favour of Hilbert mathematics depends on whether the quantum mechanics itself is accepted as fundamental. For it is only a science among many others, it cannot be privileged. For any entity is a quantum system, it is fundamental. However, even in the latter case, the fundamentality of quantum mechanics might be accepted only as to all physical entities and rejected as to mathematics.

We met again the necessity of "petitio prinicipii": The choice between Hilbert mathematics or Gödel mathematics seems to be presupposed even to our being. Each of them is postulable, but none of them is preferable or rejectable. Anyway, the existence of reasons to be preferred the one, though unknown until now, cannot be also refused.

Indeed, the Schrödinger equation is not too difficult to be interpreted in terms of an information equation in the sense above:

(1) The Planck constant might equate the physical quantity of action to information, for the latter is physically dimensionless and possesses a natural quant, a bit: the quantity of information cannot be less than a bit ever.

(2) Since transfinite series are used, the physical action of those would turn out to be also transfinite and thus inconvenient immediately. One should utilize the physical action per a unit of the length of series. The corresponding physical quantity might be identified as 'time', and the physical action per a unit of series as 'energy'. Indeed, the Schrödinger equation is dimensioned in energy and thus refers to the equation of energies. Consequently, that fact is also consistent to the interpretation of the Schrödinger equation as an information equation.

(3) The transfinite segments of bits per a unit of segment should not be distinguishable from the finite ones. Consequently, the utilization of energy identifies the cases of bits and transfinite bits. One can speak of bits generally meaning both classical and transfinite bits once their energy equivalent is used.

(4) The Schrödinger equation compares a kind of energy in its left part to the sum of two kinds of energy in its right part. These two kinds of energy in the right part correspond to the kinetic and potential energy in classical mechanics, and their sum to the total mechanic energy. The left part is dimensioned as energy, too, but it has not any correspondence in classical mechanics.

(5) That new kind of energy is proportional to the first time derivative of the wave function. The wave function itself can be considered as the characteristic function of a probability distribution and thus attachable to a certain infinite set as above. According to the equivalency of the kinds of information, the same infinite set can be represented by a transfinite series, and its time derivative interpreted accordingly as the energy of a transfinite bit or a bit for (3) above in the final analysis.

(6) A qubit is isomorphic to a usual 3D unit ball, in which two points are chosen: the one within the ball, the other on its surface (which is a unit 3D sphere). Furthermore, the latter point has to be on the circle which is perpendicular to the straight line, determined by both centre of the ball and former point. Then, that unit ball with two points chosen can be interpreted as a space ball in Minkowski space-time for some moment $t$ and with a radius $c$. $t$ ( $c$ is the velocity of light in vacuum).

(7) Any qubit depends on the square of space distance after the construction in (6), and its change would correspond to the second derivative of space distance. This is consistent both to the interpretation of information as action in (1) and to the operator $\left(\nabla^{2}\right)$ of kinetic energy in the right part of the Schrödinger equation.

(8) If the consideration is restricted to a qubit rather than to its change as in (7), the qubit determines a position in space (the space ball defined as above). That position can be interpreted as the argument of the function of potential energy, which is the other member in the sum of total mechanic energy in the right part of the Schrödinger equation.

(9) Meaning the algebraic signs in the Schrödinger equation, one can summarize a purely informational interpretation of it: the energy of a qubit is equal to the sum of the change of the energy of that qubit and the energy of the equivalent bit according the generalized sense of 'bit' in (3). In other words, the difference of information expressed in qubits and in bits is equal to its change (expressed in qubits) after information (action) is relevantly transformed in energy (action per a unit of time).

Though the Schrödinger equation admits a pure informational interpretation as above, it is consistent anyway to the hypothesis for Gödel mathematics separating mathematics from reality. To reject the latter, one should manage to demonstrate somehow that wave function can be attached to consciousness as well ${ }^{7}$, and the Schrödinger equation expresses in fact the fundamental relation of consciousness and reality or even a more fundamental essence. That more fundamental essence might be identity of any entity as a whole or as consisting of parts just right another idea of Husserl: the formal doctrine about parts and whole: Division 3 in Volume 2 of "Logical Investigations" [5], [88].

The problem of quantum mechanics to unify the discrete and continuous motions and that of philosophy to unify the discrete leap between consciousness and reality and continuous transition between them seem to share simile and even maybe one and the same structure expressible informationally by the Schrödinger equation.

\section{CONCLUSIONS \& FUTURE WORK}

The principle of universal mathematizability refers to any scientific theory as it is always exhibited in a natural language as a text. Even the texts of a more extended class are mathematizable, but this is out of the scope of the present consideration. The term of scientific theory is used in the sense of internal consistency of the text and thus includes not only the theories confirmed by experience and experiments, but also all scientific hypotheses not yet corroborated or already rejected.

\footnotetext{
${ }^{7}$ All conceptions of "quantum mind" [86], [87] imply the same or similar of that.
} 
The principle states that both a relevant and exhausted mathematical structure can be chosen for any scientific theory and that choice can be constructive.

Investigating an eventual sketch for proving it, a series of interesting circumstances appear:

First of all, the principle seems to be rather a most fundamental axiom referring to the relation of mathematics and reality. Consequently, it cannot be proved or refused in a proper sense. What is possible is to be demonstrated that it does not lead to any contradiction or to construct a model of the mathematics accepting it in the mathematics rejecting it.

The way is by means of elucidating the mismatch of (Peano) arithmetic and set theory in relation to infinity in the foundation of mathematics. Peano arithmetic does not admit infinite natural number unlike set theory postulating infinite sets. Gödel unresolvable statements appear of the stick between them.

Further, one can show that any statement of that kind correspond to some non-mathematizable scientific theory. Therefore, the principle of universal mathematizabilty is inconsistent to the Gödel incompleteness theorems (1931) [82]. Anyway, it has already demonstrated that Gödel proof implicitly accepts reality external to mathematics, and the existence of unresolvable statements is not more than a new, but equivalent expression of that assumption, which is right modelled by the relation of Peano arithmetic and set theory.

The mentioned relevant historical fact allows for the metamathematical postulate about reality as external to mathematics to be assigned to those theorems, and the kind of mathematics sharing it to be called Gödel mathematics.

On the contrary, if Peano arithmetic is generalized by replacing the axiom of induction by that of transfinite induction, the mismatch in question disappear as well as the Gödel unresolvable statement as Gentzen's proof (1936) $[80,83]$ can be interpreted in particular.

The present paper follows another pathway grounded on Husserl's phenomenology and "bracketing reality" to achieve the generalized arithmetic equivalent to that just above and necessary for the principle to be founded in alternative ontology, in which there is no reality external to mathematics, but reality is included within mathematics. That latter mathematics is able to self-found itself and can be called Hilbert mathematics in honour of Hilbert's program for selffounding mathematics on the base of arithmetic.

The principle of universal mathematizability is consistent to Hilbert mathematics, but not to Gödel mathematics. Consequently, its validity or rejection would resolve the problem which of the two mathematics refers to our being; and vice versa: the choice between them for different reasons would confirm or refuse the principle as to the being.

A few directions for future work can be:

(1) A rigorous formal proof of the principle as an independent axiom.

(2) The further development of information ontology consistent to both kinds of mathematics, but much more natural for Hilbert mathematics.

(3) The development of the information interpretation of quantum mechanics as a mathematical one for information ontology and thus Hilbert mathematics.

(4) The description of consciousness in terms of information ontology. 


\section{REFERENCES}

[1] Russell, B. The Principles of mathematics. Cambridge: Univ. Press (1903).

[2] Zermelo, E. Beweis, dass jede Menge wohlgeordnet werden kann. Mathematische Annalen 59 (4): 514-16 (1904).

[3] Brentano, F. Die Psychologie des Aristoteles: Insbesondere seine Lehre vom nous Poiētikos. Mainz: Franz Kirchheim (1867).

[4] Brentano, F. Psychologie vom empirischen Standpunkt. Leipzig: Dincker \& Humblot (1874).

[5] Husserl, E. Logische Untersuchungen. I. Leipzig: Veit \& Comp; II. Halle: Max Niemayer (1900; 1901).

[6] Husserl, E. Philosophie als strenge Wissenschaft. Logos 1(3): 289341 (1910).

[7] Husserl, E. Ideen zu einer reinen Phänomenologie und phänomenologischen Philosophie: Buch 1, Allgemeine Einführung in die reine Phänomenologie. Jahrbuch für Philosophie und Phänomenologische Untersuchungen 1(1): 1-323 (1913).

[8] Husserl, E. Vorlesungen zur Phänomenologie des inneren Zeitbewusstseins. Halle: Max Niemayer (1928)

[9] Husserl, E. Phenomenology. [Edmund Husserl's article for Encyclopaedia Britannica (1927)]

[10] Law, H. C. et al. Attention to Intention. Nature 303(5661): 12081210 (2004).

[11] Stapp, H. P. Attention, intention, and will in quantum physics. Journal of Consciousness Studies 6(8-9) 143-164 (1999).

[12] Pascual-Leone, J. Intension, Intention, and Early Precursors of Will: Constructive Epistemological Remarks on Lewis's Research Program. Psychological Inquiry 1(3): 258-260 (1990).

[13] Husserl, E. Über den Begriff der Zahl: psychologische Analysen. Halle: Heynemann (F. Beyer) (1887).

[14] Husserl, E. Philosophie der Arithmetik: Psychologische und logische Untersuchungen, Bd. 1. Halle - Saale: C. E. M. Pfeffer (R. Stricker) (1991)

[15] Heidegger, M. Sein und Zeit. Halle: Max Niemeyer (1927).

[16] Crowel, S. G. Husserl, Heidegger, and the Space of Meaning. Paths to Transcendental Phenomenology. Evanston, IL: Northwestern University Press (2001)

[17] Wendt, A. Quantum Mind and Social Science. Cambridge: Univ. Press (2015).

[18] Stapp, H. P. A quantum theory of the mind-brain interface, in Mind, Matter, and Quantum Mechanics, Berlin: Springer, 145172 (1993)

[19] Stapp, H. P. A quantum-mechanical theory of the mind-brain connection, in: Beyond Physicalism. Lanham: Rowman and Littlefield, 157-193 (2015)

[20] Dedekind, R. Was sind und was sollen die Zahlen? Braunschweig: Friedrich Vieweg \& Sohn (1888).

[21] Dotterer, R. H. The Definition of Infinity: The Journal of Philosophy, Psychology and Scientific Methods 15(11) 294-301 (1918).

[22] Skolem, T. Einige Bemerkungen zur axiomatischen Begründung der Mengenlehre. Matematikerkongressen i Helsingrofs den 4-7 Juli 1922, Den femte skandinaviska matematikerkongressen, Redogörelse, Helsinki: Akademiska-Bokhandeln, 217-232 (1923).

[23] Bohr, N. Wirkungsquantum und Naturbeschreibung. Naturwissenschaften 17(26): 483-486 (1929).

[24] Brody, N., Oppenheim, P. Application of Bohr's Principle of Complementarity to the Mind-Body Problem. The Journal of Philosophy 66(4): 97-113 (1969).

[25] Husserl, E. Einleitung in die Logik und Erkenntnistheorie. Vorlesungen 1906/07. Husserliana XXIV. Dordrecht: Martinus Nijhoff (1984).

[26] Husserl, E. (1987). Aufsätze und Vorträge (1911-1921). Husserliana XXV. Dordrecht, Martinus Nijhoff (1987).

[27] Heidegger, M. Über den Humanismus. Frankfurt am Main: Klostermann (2000).
[28] Heidegger, M. Brief über den Humanismus. In: Wegmakrken. Frankfurt AM: Klosterman, 313-364 (1976).

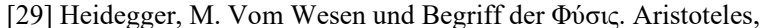
Physik B, 1. In: Wegmakrken. Frankfurt AM: Klosterman, 239301 (1976).

[30] Frege, G. Über Sinn und Bedeutung. Zeitschrift für Philosophie und philosophische Kritik, NF 100(1): 25-50 (1892).

[31] Gadamer, H.-G. Wahrheit und Methode. Grundzüge einer philosophischen Hermeneutik. Tübingen: Mohr (1960)

[32] Laverty, S. M. Hermeneutic Phenomenology and Phenomenology. A Comparison of Historical and Methodological Considerations. International Journal of Qualitative Methods 2(3): 21-35 (2003).

[33] Heidegger, M. Vom Wesen der Wahrheit. In: Wegmakrken. Frankfurt AM: Klosterman, 177-201 (1976).

[34] Heidegger, M. Erläuterungen zu Hölderlins Dichtung. Frankfurt AM: Klostermann (1951).

[35] Derrida, J. L'écriture et la difference. Paris: Seuil (1967).

[36] Derrida, J. De la grammatologie. Paris: Minuit (1967).

[37] Ricœur, P. La métaphore vive. Paris: Seuil (1975).

[38] Schrödinger, E. Die gegenwärtige situation in der Quantenmechanik. Die Naturwissenschaften 23(48): 807-812; 23(49): 823-828; 23(50): 844-849 (1935).

[39] Sartre, J.-P. L'existentialisme est un humanism. Paris: Nagel (1946).

[40] Heidegger, M. Identität und Differenz. Pfullingen: Günter Neske (1957).

[41] Seeburger, F. F. (1975) Heidegger and the Phenomenological Reduction. Philosophy and Phenomenological Research 36(2): 212-221 (1975).

[42] Morrison, J. C. Husserl and Brentano on Intentionality. Philosophy and Phenomenological Research 31(1): 27-46 (1970).

[43] Husserl, E. Cartesianische Mediationen (1929). Cartesianische Meditationen Und Pariser Vorträge. Husserliana. Bd. 1. Den Haag: M. Nijhof (1950).

[44] MacDonald, P. S. Descartes and Husserl. The Philosophical Project of Radical Beginnings. Albany: State University of New York Press (2000).

[45] Ricœur, P. Husserl: An Analysis of His Phenomenology (Northwestern University Studies in Phenomenology and Existential Philosophy). Evanston: Northwestern University Press, (1967).

[46] Henry, M. C'est moi la Vérité. Pour une philosophie du christianisme. Paris: Seuil (1996).

[47] Marion, J.-L. Dieu sans l'être. Paris: Fayard (1982).

[48] Overgaard, S. Epoché and Solipsistic Reduction. Husserl Studies 18 (3): 209-222 (2002).

[49] Mahnke, D. From Hilbert to Husserl: First Introduction to Phenomenology, Especially That of Formal Mathematics. Studies in History and Philosophy of Science (Part A) 8 (1):71-84 (1977).

[50] Tieszen, R. L. Mathematical Intuition: Phenomenology and Mathematical Knowledge. Dordrecht, etc.: Kluwer Academic, (1989).

[51] Seidel, G. J. Martin Heidegger and the pre-Socratics: an introduction to his thought. Lincoln, NE: University of Nebraska Press (1964).

[52] Iamblichus. The life pf Pythagoras. Krotona - Hollywood, CA: Theosophical Publishing House (1918).

[53] Gorham, G., Hill, B., and Slovwik. E. The language of nature. Reassessing the mathematization of natural philosophy in the seventeenth century. Minneapolis : University of Minnesota Press (2016).

[54] Hardy, L. Phenomenology of natural science (Series: Contributions to phenomenology, v. 9) Dordrecht; Boston : Kluwer Academic Publishers (1992).

[55] Aukstuolyte, N. The Interrelationship between the Mathematization of Science and the Idea of Universal Formal Language of Science. European Scientific Journal 10(12): 442452 (2014). 
[56] Wilner, J. The Mathematization of Nature: Galileo, Husserl, Mandelbrot. In The Yearbook of Comparative Literature 58: 235 240 (2012).

[57] Quine, W. V. Success and Limits of Mathematization. In: Der 16. Weltkongress für Philosophie Volume 1: 439-446 (1983)

[58] Pawlowski, T. The Limits of Measurement and of Mathematization in the Social Sciences. In: Der 16. Weltkongress für Philosophie Volume 2: 439-446 (1983).

[59] Lenhard, J., Otte, M. Two Types of Mathematization. Philosophical Perspectives on Mathematical Practice (Texts in philosophy, 12) London: College Publications, 1018-1025 (2010).

[60] Apostolatos, N. Informatics and the Internal Necessity for the Mathematization of the Sciences. In: Applied Mathematics and Parallel Computing. Heidelberg: Physica, 1-10 (1996).

[61] Roux, S. Forms of Mathematization (14th -17 th Centuries). Early Science and Medicine 15(4/5): (2010), pp. 319-337 (2010)

[62] Monteiro, S The Search for the Mathematization of the Social Disciplines. In: The Application of Mathematics to the Sciences of Nature: Critical Moments and Aspects. New York: Springer, $245-$ 252 (2002).

[63] Born, M. Physical Aspects of Quantum Mechanics. Nature 119 (2992): 354-357 (1927)

[64] Conway, J., S. Kochen. The Free Will Theorem. Foundations of Physics 36(10): 1441-1473 (2006).

[65] Conway, J., S. Kochen. The Strong Free Will Theorem. Notices of the American Mathematical Society 56(2): 226-232 (2009).

[66] Neumann, J. Mathematische Grundlagen der Quantenmechanik. Berlin: Springer, 167-173 (1932).

[67] Kochen, S. and Specker, E. The Problem of Hidden Variables in Qunatum Mechanics. Journal of Mathematics and Mechanics 17(1): 59-87 (1968).

[68] Husserl, E. Die Krisis der europäischen Wissenschaften und die transcendentale Phänomenologie: Eine Einleitung in die phänomenologische Philosophie. Philosophia (Belgrad), 1: 77-176 (1936).

[69] Sörensen, D. Theory formation and the study of literature. Amsterdam: Rodopi (1987).

[70] Zermelo, E. Untersuchungen über die Grundlagen der Mengenlehre I. Mathematische Annalen 65(2): 261-281 (1908).

[71] Popper, K. Logik der forschung: zur erkenntnistheorie der modernen naturwissenschaft. Wien: Springer (1935).

[72] Weber, M. Die 'Objektivität' sozialwissenschaftlicher und sozialpolitischer Erkenntnis. In: Archiv für Sozialwissenschaft und Sozialpolitik 19(1): 22-87 (1904).

[73] Winfired, N. Charles S. Peirce's Theory of Information: A Theory of the Growth of Symbols and of Knowledge. Cybernetics \& Human Knowing 19(1-2) 137-161 (2012)

[74] Peirce, C.S. The Regenerated Logic. The Monist 8(1): 19-40 (1896).

[75] Peano, I. Arithmetices principia: nova method exposita. RomaeFlorentiae: Fratres Bocca (1889).

[76] Lambalgen, M. van. Independence, Randomness and the Axiom of Choice. The Journal of Symbolic Logic 57(4) (1992).

[77] Kingman, J. F. C. Random Discrete Distributions. Journal of the Royal Statistical Society Series B (Methodological), 37(1): 1-22 (1975).

[78] Rozenfeld, B. A. A History of Non-Euclidean Geometry: Evolution of the concept of a geometric space. Berlin, etc.: Springer (1988).

[79] Palermo, J. Apodictic Truth: Husserl's Eidetic Reduction versus Induction. Notre Dame Journal of Formal Logic 19(1): 69-80 (1978).

[80] Gentzen, G. Die Widerspruchsfreiheit der reinen Zahlentheorie. Mathematische Annalen 112(1): 493-565 (1936).

[81] Gentzen, G. Beweisbarkeit und Unbeweisbarkeit von Anfangsfällen der transfiniten Induktion in der reinen Zahlentheorie. Mathematische Annalen 119(1): 140-161 (1943).
[82] Gödel, K. Über formal unentscheidbare Sätze der Principia mathematica und verwandter Systeme I. Monatshefte der Mathematik und Physik, 38(1): 173-198 (1931).

[83] Gentzen, G. Die gegenwärtige Lage in der mathematishen Grundlagenforshung. Forshungen zur Logic und zur Grundlegung der exacten Wissenshaften, Neue Folge 4, 5-18 (Leipzig: Hirzel, 1938).

[84] Hilbert, D. Die Grundlagen der Mathematik. Abhandlungen aus dem Seminar der Hamburgischen Universität, 6: 65-85 (1928).

[85] Einstein, A., Podolsky, B. Rosen, N. Can Quantum-Mechanical Description of Physical Reality Be Considered Complete? Physical Review 47(10): 777-780 (1935)

[86] Mindel, A. Quantum mind: the edge between physics and psychology. Portland, OR: Lao Tse Press (2000).

[87] Penrose, R. Shadows of the mind: a search for the missing science of consciousness. Oxford; New York: Oxford University Press (1994).

[88] Casari, E. On Husserl's Theory of Wholes and Parts. History and Philosophy of Logic 21(1): 1-43 (2000). 